\title{
ESTADO DE LA CUESTIÓN SOBRE LA SUBSISTENCIA DEL MUSTERIENSE DE LA VERTIENTE MEDITERRÁNEA DE LA PENÍNSULA IBÉRICA
}

\author{
JOSÉ YRAVEDRA SÁINZ DE LOS TERREROS
}

\begin{abstract}
Este trabajo supone un estado de la cuestión sobre la zooarqueología del Musteriense mediterráneo. Para ello se han analizado los datos procedentes del NR, el MNI, los perfiles esqueléticos y los patrones de alteración ósea con el fin de dilucidar las estrategias subsistenciales de los grupos humanos de este momento en esta región.
\end{abstract}

In this paper the Mousterian Archaeozoology of the Mediterranean during the Iberian Palaeolithic is discussed. The data obtained from the NR, the MNI, skeletal profiles and alterations to bone patterns have been studied in order to discover the subsistence strategies used by human groups in this region.

\section{INTRODUCCIÓN}

El análisis de esta región implica una extensa franja geográfica que se distribuye desde el norte de Cataluña hasta Andalucía oriental. La cantidad de yacimientos que se han encontrado ha sido bastante grande, pero en este trabajo sólo nos referiremos a aquellos que han tenido análisis zooarqueológicos, suponiendo un total de 24 yacimientos divididos en 76 niveles (Fig. 1).

El presente estudio pretende comparar las estrategias subsistenciales de toda la franja mediterránea. Para ello se analizará en una primera parte los patrones taxonómicos con el fin de dilucidar qué especies son aportadas a los yacimientos y cuáles son las más abundantes. Como nuestro estudio pretende centrarse en los animales aportados por los seres humanos, se prestará especial atención a aquellos taxones más susceptibles de consumo humano, como los cérvidos, los équidos, los grandes bóvidos y en última instancia a los pequeños bóvidos (a partir de ahora cápridos). Por otro lado la presencia de otros animales como los carnívoros también se considerarán por las implicaciones tafonómicas y paleoecológicas que conllevan, ya que están directamente relacionados con la competencia por los recursos con el hombre.

Los métodos usados en los perfiles taxonómicos son el número de restos $(N R)$, y el número mínimo de individuos $(M N I)$, ya que son los métodos utilizados más frecuentemente en la literatura zooarqueológica. En total son 24 yacimientos y 76 niveles los que cuentan

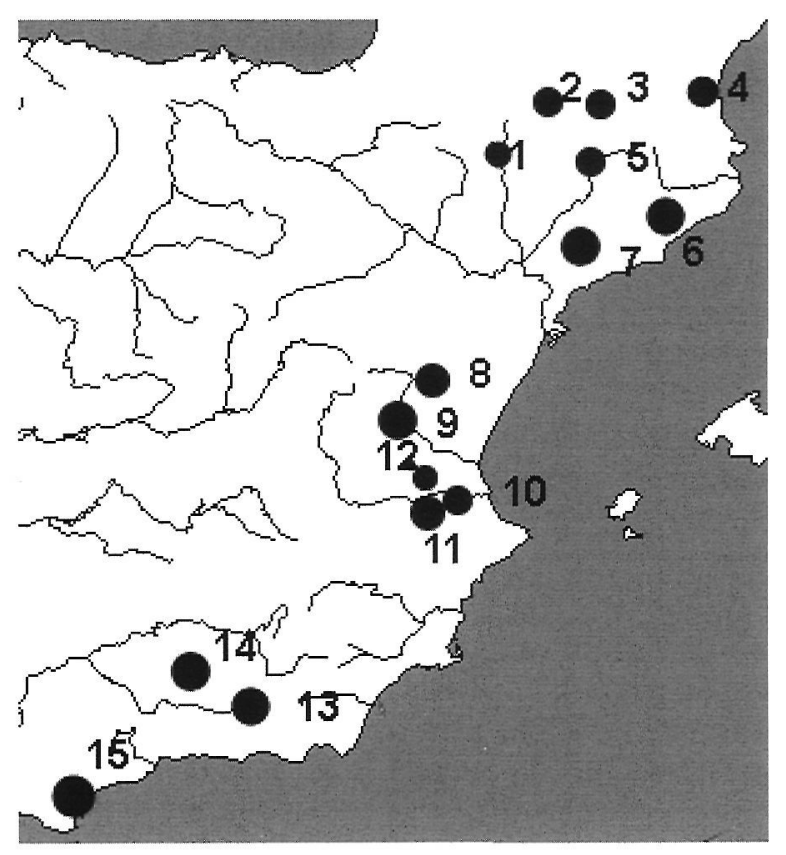

Figura 1: Situación Geográfica de los Yacimientos tratados en el texto: L'Estret de Tragó (Castañeda, 1999) (1), Cova 120 (Estévez, 1979; Rueda 1993) y Els Ermitons (Estévez, 1979, 1980) (2), L'Arbreda (Estévez, 1979, 1987; Rueda, 1993) y Mollet (Estévez, 1979, Rueda, 1993) (3), Cau del Duc de Torroella (Estévez, 1979, 1980) (4), Toll y Teixoneres (Estévez, 1979) (5), Abric Romaní (Cáceres, 1995) (6), Gegant y Muscle (Estévez, 1979) (7), San Antón (Sarrión, 1990) (8), Fuente de San Luis (Fernández y Martínez, 1989) (9), Cova Beneito (Martínez, 1996) (10), Moli Mató y Salt (Sarrión, 1990) (11), Cova Negra (Pérez Ripoll, 1977; Martínez, 1996)(12), Cova Hora (Martin, 1986) (13), Boquete de Zafarraya (Barroso et alii, 1983) (14), Gorham's Cave (Barton, 1988) (15). 
con el análisis del $N R$ y de 9 asentamientos con sus 17 niveles para el MNI.

La segunda parte de este trabajo consistirá en dilucidar qué estrategias de subsistencia siguieron los grupos musterienses. Para ello se recurrirá a los patrones de representación anatómica y a los tafonómicos. Los perfiles esqueléticos no se han analizado en muchos sitios, de esta forma, el ciervo es el animal que presenta un mejor análisis ofreciendo el cálculo de estos perfiles en 21 niveles. Para los demás taxones la menor representatividad de las muestras óseas y la escasez de yacimientos con este tipo de análisis incapacita cualquier tipo de conclusión, al no haber muchos datos. Por último y en relación con estos perfiles anatómicos se hace referencia a los estudios tafonómicos ya que como han visto algunos autores, son los más susceptibles de mostrar la responsabilidad de los diferentes agentes en las acumulaciones osteológicas (Domínguez Rodrigo, 1996, 1997a, 1997b, 1999; Domínguez Rodrigo y De la Torre, 1999; Marean, 1998).

\section{PATRONES TAXONÓMICOS}

Como puede observarse en las figuras 1 y 2 son los yacimientos catalanes los que presentan un mayor número. Sin embargo, la cantidad de niveles descritos y analizados zooarqueológicamente por Pérez Ripoll (1977) y Martínez (1993) en Cova Negra hacen del País Valenciano el lugar con mayor número de niveles tratados. En la figura 2 se han agrupado en 3 columnas diferenciando los datos procedentes de diferentes estudios y excavaciones. Entre los yacimientos catalanes sólo el Abric Romani ha ofrecido una amplia cantidad de niveles, pero tan sólo se han estudiado el E, H, I, J y K, aunque Estévez (1979) ya estudio algunos materiales de las excavaciones iniciales. En el extremo sur de la Península Ibérica sólo la Carigüela y Gorham's Cave han ofrecido un amplio número de niveles, pero en el primer caso no hay un estudio zooarqueológico propiamente dicho y del segundo sólo algunas unidades estratigráficas han mostrado un número de restos óseos representativo.

Además de los asentamientos de la figura 1 y 2 hay otros que no han sido considerados por carecer de estudios zooarqueológicos desarrollados. Algunos de estos son la cueva de las Grajas (Málaga), donde Montes Bernárdez (1991) documentó cierto predominio de ciervo seguido de cabra, caballo y Bos, en Zájara II, (Málaga) también destaca el ciervo (Cacho, 1983). En Colomera (Granada) destaca el caballo y el ciervo (Toro y Almohalla. 1981), en las Yedras (Ruiz Bustos 1978) ha documentado restos craneales y apendiculares de caballo, Bos y otros restos de zorro, ciervo, corzo y gamo y en la Carigüela junto a los équidos y cérvidos también están los carnivoros (Bouchud, 1969). En las cuevas murcianas de los Pescadores son importantes el caballo y el ciervo y en Perneras, unas especies $u$ otras varían según el nivel. Así en el estrato 6 el conejo es el taxón principal seguido de caballo, cabra y ciervo. En el 5 sobresale la cabra, luego el caballo y el ciervo, y en el 8 y 9 las principales especies son el ciervo y el jabali (Montes Bernárdez, 1985, 1988, 1991). En la Cueva del Niño predomina el caballo, el uro, el rinoceronte y el oso (Serna, 1997) y en la cueva murciana de Cueva Negra y la Sima de las Palomas destacan junto a Equus, Bos y Cervus el Megaceros (Gibert, 1996). En los yacimientos castellonenses de Cabezo Negro sólo destaca el caballo y en el Tossal de la Font, Gusi et alii $(1980,1984)$ han registrado pocos restos óseos craneales pertenecientes a los ungulados clásicos y a los carnívoros. Por último en algunos yacimientos catalanes como en la Cueva de L'Estret de Tragó destaca el ciervo, los grandes bóvidos y la cabra (Mora et alii, 1992).

Resumiendo, los datos aislados de todos estas estaciones indican que los restos óseos de dichos lugares son conjuntos muy fragmentarios de elementos craneales y diafisiarios que taxonomicamente muestran un predominio de ciervos y caballos acompañadas de otros taxones con una representación variable como carnívoros, grandes y pequeños bóvidos y megafauna.

En los yacimientos con un mayor NR son los lagomorfos los animales más representativos. Sin embargo, como han mostrado los análisis tafonómicos de Pérez Ripoll (1977), Estévez (1987) y Martínez (1996) su presencia está más ligada a causas naturales que al aporte antrópico, por esta razón no se los ha incluido en la figura 2. Sólo en Gegant, Teixoneres, Toll, Mollet, Olopte, Els Ermitons, el Romaní y el Boquete de Zafarraya el conejo no es el taxón predominante en NR. Los carnívoros también están muy bien representados especialmente entre los yacimientos catalanes a excepción del Abric Romaní y en algunos del Pais Valenciano, esto ha hecho 


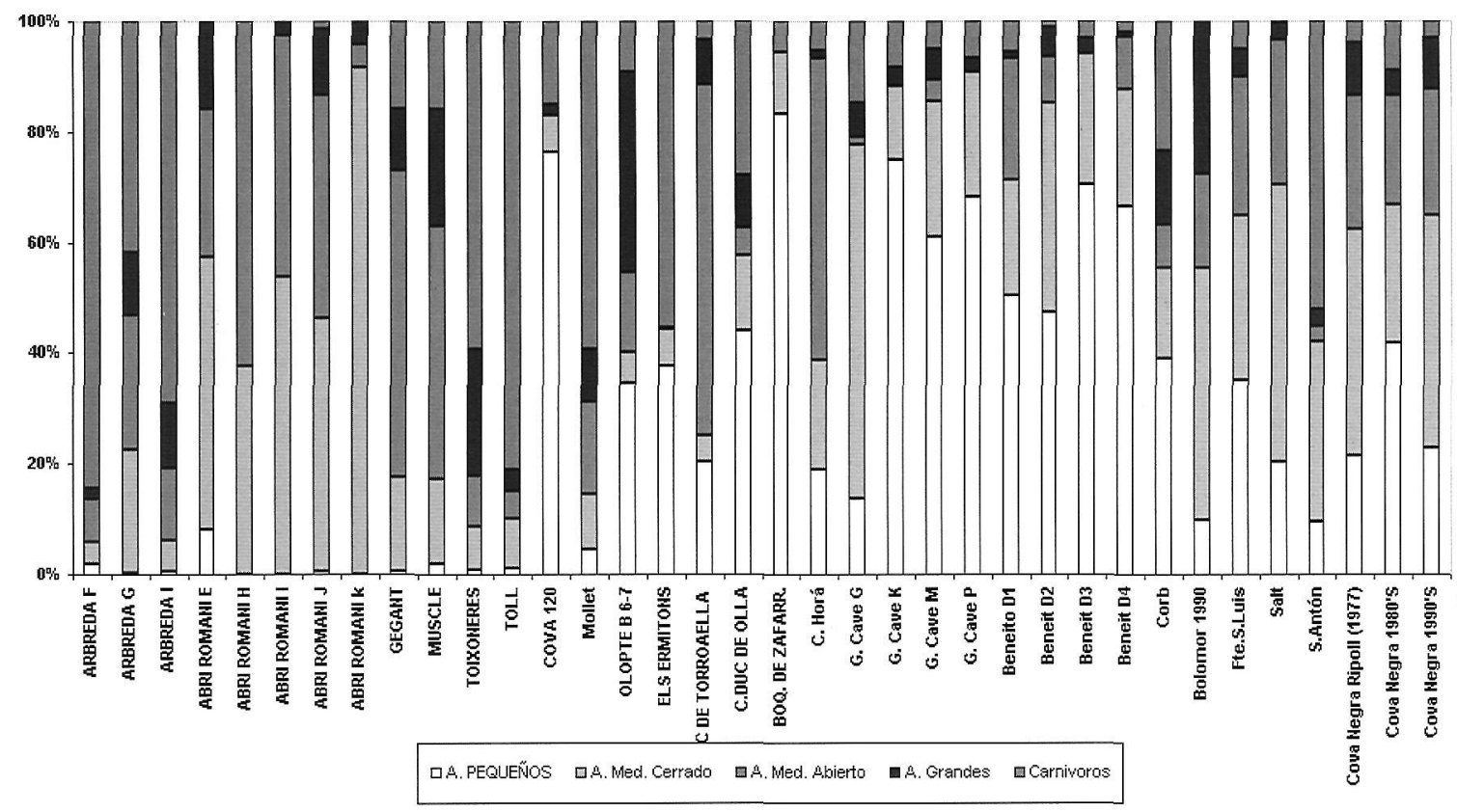

Figura 2: Patrones de Representación Taxonómica según el NR. Aparecen las especies por grupos y no se ha incluido a los lagomorfos por ser un taxón de origen biológico. En algunos casos como Cova Beneito, Gorham's Cave o Cova Negra con un gran número de niveles, se han agrupado bajo uno sólo, para poderlos observar conjuntamente en la figura. De todas formas si se quiere consultar la proporción inicial, se puede acudir a las fuentes reseñadas en la bibliografía del pie de la figura 1.

dudar de la autoría humana de algunos lugares como la Cova 120, Gegant, Mollet o algunos niveles de L'Arbreda (Rueda, 1983) o Moli Mato entre los del País Valenciano.

Si nos fijamos en los ungulados, observamos que el ciervo y los animales de talla mediana de biotopos cerrados predominan en L'Arbreda G, el Abric Romaní E, I, J, K, el Toll, $y$ en los niveles de Beneito D3, el Corb, Bolomor, el Salt, San Antón, Cova Negra (Pérez Ripoll, 1977), Cova Negra 12, 10, 9 y 3, Cova Negra V (Martínez, 1996) y en Gorham's Cave $\mathrm{G}, \mathrm{R}, \mathrm{T}$, abarcando un total de 11 yacimientos y 19 niveles, sin alcanzar en casi ningún caso el $65 \%$ de los restos, y por tanto, sin llegar a alcanzar especialización (Fig. 2).

Los animales de tamaño medio de espectros abiertos entre los que destacan los équidos destacan en L'Arbreda F, el Romaní H, el Gegant, Muscle, Mollet, Cau del Duc de Torroella, Cova Hora 1-61, Cova Negra VI (Martínez, 1996), Cova Negra 14, 13, 11, 6 y San Antón lo que equivale a 11 yacimientos y 15 niveles. En ocho de los cuales supera el 60 $\%$ de los restos si se excluye a los carnívoros, en concreto, en los casos de San Antón, Cova Horá, y Cau del Duc de Torroella, Gegant, L'Arbreda F, Muscle y Mollet (Fig. 2).

Bos es el principal taxón en Teixoneres (1-2) y L'Arbreda (Musteriense) según Rueda (1993) aunque no llega a alcanzar espe- cialización. La cabra destaca en Cova 120 , Olopte b, Els Ermitons, Cau del Duc d'Ollà, el Boquete de Zafarralla, Gorham's Cave K, M, N, P, Beneito D1, D2, D4, Molí Mató, Corb, Fuente de San Luis, Cova Negra II, IIIa, IIIb, IV (Martínez, 1996) y Cova Negra 7 superando el $60 \%$ sólo en Cova 120, Boquete de Zafarraya y Els Ermitons donde se podría pensar en cierta especialización (Fig. 2).

El predominio de cada una de las especies documentada concuerda con el entorno cercano a cada yacimiento, y en los casos en los que se da algún taxón en un medio diferente al que debiera adscribirse, como la cabra en Gorham's Cave y Cova Negra, puede deberse a la cercanía de zonas de roquedo próximas y a la mayor distancia de la costa en aquel momento.

A pesar de esto se pueden hacer ciertas diferenciaciones regionales que muestran unos patrones bien diferenciados entre los yacimientos catalanes, y los del País Valenciano y Andalucía.

En los catalanes destaca la abundancia de carnívoros y la similitud entre équidos y cérvidos que presentan casi siempre valores próximos. Después siguen los animales de talla grande que casi nunca muestran porcentajes muy altos. Por último está la escasez de animales de talla pequeña, concretamente de cabra que sólo aparece en Cova 120, Olopte, 
Els Ermitons y Cau del Duc de Torroaella. Por el contrario los yacimientos del País Valenciano muestran patrones más diversificados en los que predominan los cérvidos y los cápridos y en última instancia los équidos y grandes bóvidos. Por último en los yacimientos andaluces son los cápridos los animales principales seguidos según los casos de cérvidos y équidos.

Las causas de esta representación no puede explicarse por causas climáticas, ya que en la región mediterránea salvo en el norte de Cataluña, los cambios climáticos sólo son perceptibles por una mayor o menor suavidad del clima, esto ha favorecido que en diversos yacimientos se hayan encontrado restos de especies de medios templados como castor, macaco o hipopótamo. La explicación puede deberse a los condicionantes geográficos, así la situación en el interior de varios sitios próximos a zonas escarpadas favorecerá la presencia de los cápridos. De la misma forma la situación climática templada favorecerá también la mayor diversidad ecológica.

Junto a la presencia física de los restos de carnívoros que ya se comentó con anterioridad es importante destacar que muchas veces pueden no estar presentes físicamente pero sí a través de sus actos, por medio de marcas de diente, huesos regurgitados o coprolitos. Asi en diversos sitios han quedado patente los síntomas de su acción, como en el Abric Romaní (Cáceres, 1995, 1996a, 1996b, Cáceres et alii 1998; Aimene, 1998, Rosell, 2001; Fernández Laso 2002), en L'Arbreda, Els Ermitons, Mollet I, III y Cova 120 (Estévez, 1979, 1980; Rueda, 1993, Terradas y Rueda, 1998), en Cova Beneito, Cova Negra (Pérez Ripoll, 1977; Lindly, 1988; Villaverde y Martínez 1992; Iturbe et alii, 1993; Martínez, 1996) y Gorham's Cave (Barton 1988).

Entre los carnivoros destaca el oso junto a la hiena en los yacimientos catalanes, pero en los asentamientos del País Valenciano así como en los andaluces guardan una proporción bastante baja siendo más importantes los cánidos (Yravedra, 2000, 2001).

En lo que respecta al $M N I$ son pocos los yacimientos que cuentan con este tipo de análisis, tan sólo 9 con un total de 20 niveles, de los cuales sólo 7 ofrecen datos sobre las edades de los individuos representados. Además sus valores son muy poco representativos y no contradicen los que ofrecen el NR. Por esta razón se ha optado por basar el análisis taxonómico en el NR.
En lo referente a las edades destacan los adultos en casi todos los casos, pero los infantiles y subadultos también están bien representados. Para la cabra, Martínez (1996) documenta un predominio de adultos en Beneito y Cova Negra al contrario que en Bora Gran y el Boquete de Zafarralla. En el caso de Bos parece haber cierto equilibrio, aunque en Cova Negra tengan primacía los adultos (Martínez, 1996). En el Abric Romaní suelen darse valores bastante parejos pero en algunos niveles como en el $E$, el $K$ y el $J$ son más importantes los infantiles (Aimene, 1998; Rosell, 2001; Fernández, 2002).

Siguiendo con el análisis taxonómico es interesante ver si se puede mostrar alguna relación entre las distintas facies musteriense y la fauna asociada. Así, en los niveles con un Musteriense Charentiense Quina (Els Ermitons, Beneito D2-4, Fuente de San Luis, Salt, Bolomor y Cova Negra $14,12-9,7-6$ ) no hay una especie claramente predominante y aunque la cabra es el taxón principal en algunos sitios no implica ningún tipo de selección intencional, ya que el yacimiento en el que aparece es natural de su entorno. Para el Musteriense Típico (Boquete de Zafarralla, Cueva Horá, Gorham's Cave, Cova Negra 8, 5, 4) ocurre lo mismo y en el caso del Musteriense Final-Evolucionado (Beneito D1, Cova Negra 2-3) tampoco se pueden hacer grandes precisiones. Por lo que en principio no se puede establecer ninguna relación clara entre los tipos culturales y los faunísticos.

Desde el punto de vista cronológico se observa que los yacimientos más antiguos, como los del Riss y el Würm I tienen una mayor proporción de animales grandes o de espectro abierto. Como por ejemplo, el caballo en Cova Negra, donde alcanza valores que luego no se vuelven a dar en los momentos posteriores. En las cuevas catalanas de Gegant, Muscle, Mollet, Cau del Duc de Ullà y Teixoneres la megafauna está presente por más de un taxón. Igual ocurre en el Corb o Bolomor donde hay una gran variedad taxonómica de animales de gran tonelaje.

Entre el Würm I y el Würm II, el ciervo y la cabra (en medios próximos a zonas escarpadas) comienzan a predominar en algunos yacimientos del Würm II (Fuente de San Luis, Salt, San Antón, Cova Negra 7, 6). En los asentamientos de transición Würm I-Il se da una tendencia similar a los del Würm II, con la ligera diferencia de que los grandes bóvidos y los équidos guardan unos porcentajes mayores 
(Toll, Cova 120, Olopte b, Cau del Duc de Torroella, Cova Negra 10-8). Por lo que en el transcurso del Würm I al II, se seguiría dando una tendencia similar a la del Riss-Würm de gran abundancia de taxones de espacios abiertos y de tamaño grande, pero que ahora inician una tendencia de retroceso frente a otros animales como los ciervos y las cabras que empiezan a predominar en los lugares bajos o los altos, según sea uno u otro.

Del Würm II al 30.000 vemos cómo esta tendencia se mantiene, y así en los estratos del Würm II-III (L'Arbreda G, Cova 5-4), sigue dándose una presencia alta de caballo y de animales grandes como los grandes bóvidos y la megafauna, pero que tienen porcentajes menores en los niveles posteriores (Arbreda F, Cova Negra 3-1, los finales de Gorham's Cave, el Boquete de Zafarralla y Beneito D1-D4) a excepción de Cova Negra II. Por lo que si asumimos "que la fauna representada se debe a la acción humana" concluimos que durante todo el Musteriense se da cierta continuidad en la captación de los recursos, mostrándose cierta tendencia progresiva hacia la captura de especies de menor tamaño, que tendrá su continuidad durante el Paleolítico Superior (Yravedra, 2000, 2001). Aunque en todo el Musteriense inclusive en las fases más recientes, se sigue accediendo a animales de gran tamaño como la megafauna o los bóvidos. Sólo en los niveles más modernos de Cova Beneito y el Boquete de Zafarraya se observa una regresión importante de estos taxones.

Esta conclusión que hemos realizado al final sólo puede ser asumida si se demuestra la autoría humana de las diferentes acumulaciones óseas. La cual es demostrable a través del estudio combinado de los perfiles anatómicos y los patrones de alteración ósea. Siendo estos últimos los más resolutivos en la identificación de los diferentes agentes que pueden intervenir sobre una acumulación ósea, $e$ incluso pueden distinguir en qué orden intervienen, tal y como han demostrado varios autores (Blumenschine, 1995; Dominguez Rodrigo, 1996, 1997a, 1997b, 1999; Domínguez Rodrigo y De la Torre, 1999; Marean, 1998; Yravedra, 2000, 2001).

\section{PERFILES ANATÓMICOS Y TAFONÓMICOS}

Anteriormente nos referíamos junto a los patrones tafonómicos también al estudio de los perfiles esqueléticos. El tratamiento de los perfiles esqueléticos empezó a utilizarse en los años setenta cuando numerosos autores empezaron a preocuparse sobre el transporte de las presas, de manera que lo representado era sintomático de lo transportado. De esta forma siguiendo este planteamiento y partiendo de las observaciones etnológicas Binford y otros autores vieron que las sociedades suelen rentabilizar el transporte de las carcasas llevando hasta el lugar de consumo aquellas porciones óseas más alimenticias, por lo que una acumulación caracterizada por aquellos elementos menos nutritivos sería sintomática de un acceso secundario. De esta manera Binford (1981) y Stiner (1994) llegan a la conclusión de que los patrones de representación anatómica eran el mejor método para distinguir un acceso primario de otro secundario. De esta forma un asentamiento con mayoría de elementos axiales y apendiculares superiores se interpretaría como un acceso altamente cárnico $\mathrm{y}$, por tanto, primario; sin embargo, si la representación ósea correspondía al cráneo y los segmentos inferiores de las extremidades se interpretarían como un consumo de baja calidad cárnica y por tanto, secundario. En función de este patrón los estudios de diversos yacimientos indican estrategias secundarias para los homínidos del Paleolítico Medio, ya que sus perfiles esqueléticos ofrecen un predominio de elementos craneales y apendiculares inferiores (Binford, 1981, 1984; Stiner, 1994; Martínez, 1996). Por otro lado en algunos trabajos recientes se ha puesto de manifiesto la problemática de estas interpretaciones y otras, que tienden a identificar estrategias de transporte de carcasas durante el Paleolítico, ya que son numerosos los agentes que pueden condicionar la representación anatómica final, y por tanto su equifinalidad.

Dentro de los factores que pueden influir se puede destacar la acción destructiva de los carnívoros, las alteraciones diagenéticas o la inapropiada metodología de excavación e investigación de numerosos yacimientos trabajados en fechas tempranas anteriores a los años setenta, donde sólo se recogían aquellos elementos óseos diagnósticos como dientes y epífisis; para una mayor detalle sobre esta problemática se pueden ver los trabajos de Dominguez Rodrigo (1999) e Yravedra (2000, 2001).

En el caso de los yacimientos aquí tratados se analizarán sólo los patrones de aquellas especies más susceptibles de con- 


\begin{tabular}{|l|l|l|l|l|}
\hline YACIMIENTOS CIERVO & CR / DIEN. & TRONCO & EXT. PROX- & EXT. DIST. \\
\hline COVA NEGRA (RIPOLL 1977) & $58,8 \% / 50 \%$ & $1,4 \%$ & $6,8 \%$ & $35,2 \%$ \\
\hline COVA NEGRA IV & $62,6 \% / 50 \%$ & & $12,8 \%$ & $23,3 \%$ \\
\hline A.ROMANI I & $31 \% / 15,5 \%$ & $16,6 \%$ & $34,4 \%$ & $18,9 \%$ \\
\hline A. ROMANI J & $18 \% / 11 \%$ & $16 \%$ & $33 \%$ & $23 \%$ \\
\hline A. ROMANI K & $16 \% / 10 \%$ & $14 \%$ & $30 \%$ & $34 \%$ \\
\hline
\end{tabular}

Tabla 1: Patrones de Representación Anatómica en ciervo.

sumo humano, como son los cérvidos, los équidos, los grandes bóvidos y los cápridos, pero sólo se tratarán los niveles que tengan muestras más representativas.

Para el tratamiento de los perfiles anatómicos no hay muchos niveles y los que hay son poco significativos. Así hay 15 yacimientos y 21 niveles que muestran este tipo de análisis, pero de ellos sólo el nivel I de L'Arbreda y los E y K del Abric Romaní superan el centenar de restos. A excepción de estos niveles en los demás casos predominan los elementos craneales y apendiculares inferiores, auque se observa una curiosidad que no tiene por qué ser casual. Sólo los casos del Abric Romaní, L'Arbreda, Cova Beneito y Cova Negra presentan restos apendiculares superiores, Io cual puede tener alguna relación con las fechas de sus excavaciones en los años 80-90.

Otra característica documentada en estos cuatro yacimientos es la acción de los carnívoros, que han podido influir en la alteración del registro (Pérez Ripoll, 1977; Villaverde y Martínez 1992; Iturbe et alii, 1993; Cáceres, 1995, 1996a, 1996b; Martínez, 1996; Cáceres et alii, 1998; Rosell 2001, Fernández, 2001). De todos los niveles sólo se han seleccionado aquellos más representativos (Tabla 1), en los cuales destaca la sobrerepresentación de los elementos craneales y distales a excepción del nivel I, J y $\mathrm{K}$ del Abric Romaní donde los proximales aumentan. Por otro lado debido a las alteraciones sufridas por los carnívoros no se puede especificar qué patrones de transporte se siguieron en el aporte de las presas.

Para Bos sólo hay 5 niveles, los cuales muestran tan sólo restos apendiculares distales y craneales, aunque como en el caso anterior los niveles del Abric Romaní también presentan elementos apendiculares superiores (Cáceres, 1996 a, b, Rosell, 2001; Fernández 2002).

En el caso de los équidos, sólo se han analizado los perfiles esqueléticos en cuatro yacimientos y siete niveles pero dada su escasa representatividad no permite extraer conclusiones significativas. Solamente puede decirse que en Cueva Horá y el Abric Romaní predominan los restos craneales y apendiculares distales. Igual que en Cova Beneito y Cova Negra, aunque aquí los restos proximales están algo representados.

Para Rupicapra apenas se puede sacar ninguna conclusión ya que tan sólo hay un estrato con tres restos. En cambio la cabra aparece en 17 niveles, sobrepasando el centenar de restos en 4 niveles. En Cueva Horá, en los niveles con menos restos siguen predominando los restos craneales y distales, aunque ahora el esqueleto axial y las secciones apendiculares proximales aparecen documentadas. En Cova Beneito se da el mismo fenómeno aunque entre los restos proximales abundan las partes traseras. En los niveles de Cova Negra con pocos restos, sólo se conservan elementos apendiculares inferiores. Tan sólo el nivel 3A muestra una dispersión variada con predominio de cabeza y segmentos inferiores, pero en el que el esqueleto axial y el apendicular superior están bien representados. Si nos fijamos en la tabla 2 y observamos los 4 niveles que superan los 90 restos se aprecia un predominio de las porciones inferiores y las craneales.

Tras ver los perfiles anatómicos sólo queda referirse a los estudios tafonómicos. Ya se comentó algo sobre la importancia taxonómica de los lagomorfos en gran cantidad de sitios y de su vinculación con diferentes

\begin{tabular}{|l|l|l|l|l|}
\hline YACIMIENTOS CABRA & CR / DIEN. & TRONCO & EXT. PROX- & EXT. DIST. \\
\hline COVA NEGRA (RIPOLL 1977) & $58 \% / 56 \%$ & $3 \%$ & $9 \%$ & $29 \%$ \\
\hline COVA NEGRA 3B & $26 \% / 18,9 \%$ & $7,8 \%$ & $26 \%$ & $40,2 \%$ \\
\hline COVA NEGRA 2 & $35 \% / 21 \%$ & $20 \%$ & $10,4 \%$ & $35 \%$ \\
\hline BENEITO D4 & $29,6 \% / 20 \%$ & $5,5 \%$ & $25 \%$ & $40 \%$ \\
\hline
\end{tabular}

Tabla 2: Patrones de Representación Anatómica en cabra. 
agentes naturales (Pérez Ripoll, 1977; Estévez, 1979; Villaverde y Martínez Valle, 1992; Rueda 1993; Martínez Valle, 1996). En lo referente con los macromamíferos sólo existen algunos análisis para el Abric Romaní (Cáceres, 1995, 1996 a, b; Aimene, 1998; Rosell, 2001; Fernández, 2002), Cova Negra (Pérez Ripoll, 1977; Martínez Valle, 1996), Cova Beneito (Martínez Valle, 1996), Cova 120 (Rueda, 1993; Terrades y Rueda, 1998); Mollet y L'Arbreda (Estévez, 1979, 1987; Rueda, 1993).

En los estudios efectuados sobre los yacimientos catalanes, Estévez $(1979,1987)$ cuantifica unos cuantos restos con marcas de corte y percusión, lo que le lleva al autor a interpretar que los homínidos de estos momentos descarnaban a la presa y luego fracturaban los huesos para el consumo medular, teniendo los carnívoros la posibilidad de hacer un acceso secundario sobre determinadas secciones (costillas y epífisis). En el trabajo de Rueda (1993) sobre este mismo lugar y otros se analizan las superficies óseas, los huesos quemados y los patrones de fracturación y, se llega a la conclusión de que los patrones de fracturación son mayores en aquellos yacimientos de origen antrópico, que en los de aporte natural o de carnivoros. Así, en L'Arbreda se distinguen dos fases dentro del Musteriense. En la primera habría un mayor protagonismo de carnívoros, como indica su gran representación y el escaso número de huesos quemados. En la segunda aumentaría el papel antrópico, como indica el incremento del porcentaje de huesos quemados, la reducción de los restos de carnívoros y la mayor fragmentación.

En Mollet I, se reconoce un mayor protagonismo antrópico, tal y como denota la gran fragmentación y los huesos quemados, pero se percibe también una gran incidencia de carnívoros, que frecuentaron el asentamiento, en los momentos de desocupación humana. Finalmente en Cova 120 se observa, que son los carnívoros el principal agente acumulador y los homínidos, meros merodeadores. De hecho en este yacimiento apenas hay huesos quemados, los índices de fragmentación son inferiores y las especies carnivoras predominan en la representación taxonómica. En cualquier caso, dentro de este yacimiento, Rueda, distingue diferencias, entre los niveles IV y $V$, en las que el papel humano sería mayor en el nivel más reciente (el IV), que si tiene huesos quemados. De todas la presencia de huesos alterados térmicamente no tiene por que indicar necesariamente un aporte humano, ya que pueden sufrir esta alteración en ocupaciones antrópicas posteriores a la desarrollada por los carnivoros.

En los trabajos de Cáceres (1995, 1996 a, b), Aimene (1998), Rosell (2001) y Fernández (2002) sobre diversos niveles del Abric Romani, nos trasmiten la idea de que junto a la acción humana también han actuado los carnivoros como carroñeadores. De esta forma junto a las marcas de descarnación, evisceración, desollado o desarticulado se han documentado también marcas de diente, dándose en algunos casos superposiciones de marcas de diente sobre las de corte y por otro lado la situación de coprolitos sobre los hogares indican su estancia posterior a la antrópica.

En otros asentamientos catalanes como L'Estret de Tragó, se han documentado una gran abundancia de alteraciones térmicas y fracturas en espiral, localizadas sobre huesos largos, así como puntos de impacto sobre estos huesos y otros planos y primeras falanges, indicativas de un acceso antrópico (Mora et alii, 1992).

Siguiendo la revisión de otros yacimientos Musterienses, sólo nos queda por ver los de la región valenciana de Cova Negra y Cova Beneito analizados por Martínez (1996) y Villaverde et alii (1996).

En Cova Negra, como en todos los anteriores se vuelve a repetir la asociación de humanos y carnívoros. En lo que se refiere a los materiales, se aprecia cómo los niveles con menor fragmentación de ungulados (XI b, II) coinciden con aquellos cuyas pequeñas presas tienen mayor fragmentación. De igual manera la fracturación entre los huesos de presas pequeñas difiere según las zonas, así las del sector oeste están más fragmentadas que las del sector sur, lo que puede deberse a una mayor actividad antrópica en esa parte del yacimiento (Martínez, 1996).

Desde un punto de vista secuencial se aprecia que la actividad de los homínidos es menos intensa en los niveles inferiores, los cuales tienen mayor frecuentación de carnivoros, y mayores indicios de su actividad como refleja la ausencia de elementos axiales y epífisis. Así según Martínez el aporte de ciervos en los niveles VI-IV serían de origen antrópico, frente al III a - II que sería de carnivoros.

Las marcas de origen antrópico producidas en el ciervo son muy reducidas y se ciñen a labores de desarticulación, descarnado 
y fracturación, que aparecen en todos los huesos apendiculares (proximales y distales), siendo las extremidades la sección con más número de marcas.

En la cabra la representación anatómica indica según este autor, que en los niveles III a - Il hay un aporte de carnivoros, mientras que en el nivel III b se distingue otro mixto debido a la presencia de unas marcas de carnicería y de desarticulación en algunos restos.

En el aporte de los équidos distingue un aporte de carnívoros en el nivel II, uno mixto en el III b y en el VI - V otro antrópico. Las marcas de carnívoros sólo se han detectado en los niveles II y III b en una proporción baja.

En los escasos restos de los otros ungulados se dice que el rinoceronte del estrato III a es de aporte carnívoro y el del XII de aporte humano. El jabali responde a un aporte antrópico por las marcas de carnicería que presentan.

En función de esto se pueden diferenciar dos momentos. El primero exclusivo de carnivoros comprendido por los primeros niveles, frente a un segundo de aporte antrópico. Por otro lado se diferencia un consumo de cápridos por carnivoros de otro homínido especializado en los demás ungulados (équidos y cérvidos).

En Cova Beneito, como en Cova Negra se da una gran fracturación de las diáfisis, lo que ha propiciado un gran número de niveles de restos menores a $3 \mathrm{~cm}$ (Martínez, 1996). Pero se aprecia cómo la fragmentación disminuye en aquellos niveles que no son antrópicos, por lo que se vuelve a producir la alternancia de varios agentes en la aportación del conjunto.

En el caso del ciervo, las marcas de corte y la ausencia de marcas de diente indican que su presencia se debe a la acción antrópica. A pesar de que entre los elementos óseos predominen los craneales y los distales de las extremidades. En los équidos la ausencia de marcas de diente y la presencia de marcas de corte, junto a la mayor representación de elementos axiales y proximales indican un aporte humano.

En la cabra, el predominio de marcas de carnívoros en los niveles musterienses (C2, D1D4) señala a los carnivoros como el principal agente responsable del conjunto óseo. Además, no hay ningún resto que muestre solapamientos o asociación de evidencias humanas, por lo que no hay nada que indique que los carnívoros accedieron a las cabras en un consumo secundario.
En los pocos restos de los otros ungulados, se han apreciado marcas de carnivoro en el rebeco y marcas antrópicas en el Sus. Luego hay otros como los bóvidos, el corzo o los carnívoros que no presentan ninguna evidencia antrópica (Martínez Valle, 1996).

Por lo que resumiendo se puede concluir que las evidencias de los diferentes yacimientos tienden a confirmar la alternancia de humanos y carnívoros en la ocupación de los asentamientos, aunque parece ser que según nos acercamos al Paleolítico Superior aumenta la ocupación antrópica frente a los carnívoros, que es más importante en los niveles más antiguos, así en los estratos más antiguos de Cova 120 , L'Arbreda, Cova Beneito y Cova Negra se produce un mayor protagonismo de los carnívoros frente a los de momentos más modernos de Mollet, Abric Romaní, Cova Beneito y Cova Negra. En cualquier caso la acción de los carnivoros continúa también en aquellos niveles más humanizados, por lo que el registro óseo se muestra muy alterado, tanto por estos agentes como por otros como la conservación diferencial, el shoulder effect o transporte diferencial, las labores de investigación etc. Por lo que no es posible determinar qué patrones de transporte siguieron los grupos humanos en el aprovechamiento de las carcasas. Por otro lado con los datos propuestos se puede pensar en la utilización de estrategias cinegéticas entre los grupos musterienses de esta región, ya que diferentes lugares como L'Arbreda, Abric Romaní, L'Estret de Tragó, Cova Beneito y Cova Negra reflejan un importante acceso cárnico anterior al de otros carnivoros.

\section{CONCLUSIONES}

Tras lo visto en este trabajo las conclusiones no son muy numerosas debido a que la franja geográfica es muy amplia y el número pequeño de yacimientos con estudios zooarqueológicos, y además la escasez de análisis tafonómicos no permiten hacer muchas precisiones sobre el papel antrópico en las diferentes acumulaciones osteológicas.

En función de esto hemos visto cómo taxonómicamente son los cérvidos los animales mejor representados. Sin embargo, si nos fijamos en aspectos más particulares se pueden observar ciertas diferencias regionales.

En Cataluña predominan los cérvidos y los équidos. En el País Valenciano se da un aspecto más diversificado, estando los cápridos 
y cérvidos mejor representados que los demás. En Andalucía destaca la abundancia de cápridos que sólo es comparable en Cataluña y el País Valenciano en aquellos yacimientos situados en regiones del interior próximos a zonas escarpadas. Es importante también destacar la presencia de los carnívoros que son especialmente abundantes en Cataluña, aunque en las otras regiones también han aparecido físicamente o indirectamente a través de coprolitos o marcas de diente.

Cronológicamente y "asumiendo" la autoría humana concluimos que la acción antrópica tiende a centrarse en animales de menor tamaño según avanza el Paleolítico. Sin embargo para comprobar esto hay que ver las verdaderas implicaciones humanas sobre la fauna representada.

Esto nos lleva a definir qué agentes fueron los responsables de las acumulaciones óseas. Para lo cual partimos de los perfiles esqueléticos y los tafonómicos. Los primeros resultan ser muy poco concluyentes al presentar muestras óseas profundamente alteradas por la labor investigadora especialmente en aquellos lugares excavados en fechas antiguas. Igualmente la representatividad de las muestras son muy reducidas y sólo en el ciervo y la cabra aparecen niveles con conjuntos superiores al centenar de restos, en cualquier caso la escasez de datos no permite obtener conclusiones sobre este aspecto.

Esto nos lleva al análisis de las corticales óseas, que como los anatómicos no se han estudiado en muchos casos. A pesar de ello los casos analizados permiten sacar algunas conclusiones interesantes.

En primer lugar destaca la dualidad en la ocupación de los asentamientos por carnívoros y humanos. La alta proporción de carnívoros en algunos sitios y la documentación de marcas de diente o coprolitos confirman esto. Así hay situaciones en las que el principal responsable de la acumulación son los carnívoros (Cova 120), pero en otros se ha visto cómo los carnívoros aprovechan las sobras dejadas por los humanos como en el Abric Romani. Por último en otros casos se da un patrón dual de ocupación en el que los carnivoros son el principal responsable de determinados taxones frente a los seres humanos que aportan otros (Cova Negra, Cova Beneito). En función de esto se puede ver cómo tanto humanos como carnívoros compitieron por la ocupación de ciertos asentamientos y cómo el aprovisionamiento humano de ciertos recursos cárnicos especialmente de ciervo y caballo se debió a la práctica de estrategias cinegéticas.

Por otro lado esta conclusión sólo ha podido establecerse a partir de los estudios recientes del Abric Romaní, Cova Beneito, Cova Negra, y L'Arbreda principalmente, por lo que llamamos la atención sobre la necesidad de revisar materiales y de efectuar estudios zooarquelógicos amplios que incluyan análisis tafonómicos, de tal manera que podamos incrementar los perfiles taxonómicos y anatómicos documentados con nuevos yacimientos y nuevos niveles que permitan hacer una mejor valoración de esta extensa zona geográfica.

\author{
José Yravedra Sainz de los Terreros \\ Avda. Alberto Alcocer, 47 \\ 28016 Madrid \\ joyravedra@hotmail.com
}

\section{BIBLIOGRAFIA}

AIMENE, M., 1998: "Les différents aspects de l'activité anthropique du niveau E de L'Abric Romaní Barcelona, Spain)", en Économie Préhistorique, les comportements de subsistance au Paléolithique, 193-203, XVIII Rencontres Internationales d'Archéogéologie et d'Histoire d'Antibes, Editions APDCA, Sophia Antipolis.

BARROSO RUIZ, C., GRACIA SÁNCHEZ, M. RUIZ BASTOS, A. MEDIMACARA, P. y SANCHIDRIAN TORTI, J.L., 1983: "Avance al estudio cultural antropológico y paleontológico de la cueva del Boquete de Zafarraya", Antropología y Paleoecologia Humana, 3, 3-12.

BARTON, C.M., 1988: Lithic Variability and Middle Paleolithic Behavior. New Evidence from the Iberian Peninsule, BAR International Series 408, Oxford.

BINFORD, L. R., 1981: Bones: ancient men, modern myths, Academic Press, New York.

BINFORD, L. R., 1984: Faunal Remains from Klasius River Mouth, Academic Press, New York.

BLUMENSCHINE, R.J., 1986: Early hominid scavenging opportunities. Implications of carcass availability in the Serengeti and Gnorongoro ecosystems, BAR International Series 283, Oxford.

BLUMENSCHINE, R.J., 1995: "Percussion marks, tooth marks and the experimental determinations of the timing of hominid and carnivore access to long bones at FLK Zinjanthropus, Olduvai Gorge, Tanzania", Journal of Human Evolution, 29, 21-51.

BOUCHUD, 1969: "Apéndice de la fauna de la Carigüela", L'Antropology, 63, 361-364.

CÁCERES, I., 1995: Estudios tafonómicos de los procesos de formación del Nivel l del Abric Romani, Capalledes Barcelona). La Influencia de la actividad antrópica, Tesis de Licenciatura; Departamento de historia y Geografía. Facultad de Letras, Universidad Rovira i Virgili. Inédita.

CÁCERES, I., 1996a. "Secuencia de los procesos y mecanismos de alteración de la asociación fósil de macromamiferos del Nivel I del Abric Romani (Capalledes Barcelona)". Comunicación de la ll reunión de Tafonomia y Fosilización, 73-78. 
CÁCERES, I., 1996b: "Taphonomic Processes in the Level I. Abric Romaní. Barcelona, Spain)", III International congress of prehistoric and protohistoric sciences (Forli, 8-14 Sept. 1996), Tome 2, 381-386.

CÁCERES, I., ROSELL, J. y HUGUET, R., 1998: "Séquence d'utilisation de la biomasse animale dans le gisement de l'Abric Romani (Barcelone, Espagne)", Quaternaire, 94 , 379-383.

CACHO QUESADA, C., 1983: "El yacimiento de Zájara II (Cueva de Almanzara). Historia de su investigación y análisis de su industria", Homenaje a M. Almagro Bach, 203217.

CASTAÑEDA, N., 1999: La Cova de L'Estret de Tragó. Estrategias de explotación de los recursos minerales en el Paleolítico Medio del Noroeste peninsular, Tesis de Licenciatura, Universidad Autónoma de Barcelona. Inédita.

DOMINGUEZ RODRIGO, M., 1996: "Caza y Carroñeo: Reflexiones en torno a la validez de las diagnosis aplicadas al registro arqueológico", Tabona, IX, 273-298

DOMINGUEZ RODRIGO, M., 1997a.: "Meat eating by early hominids at FLK Zinj 22 Site, Olduvay Gorge Tanzania: An experimental approach using cut-marc data", Journal of human Evolution, 33, 669-690.

DOMINGUEZ RODRIGO, M., 1997b: "A Reassesment of the study of cut marcs patterns to infer hominid manipulation of fleshed carcasses at the FLK Zinj 22 Site, Olduvay Gorge Tanzania", Trabajos de Prehistoria, 54: 2, 29-42.

DOMÍNGUEZ RODRIGO, M., 1999: "The study of skeletal part profiles: An ambiguous taphonomic tool for Zooarchaeology", Complutum 10, 15-24.

DOMÍNGUEZ RODRIGO, M. y DE LA TORRE SAINZ I., 1999: "Estado actual del debate de la caza y el carroñeo en el origen del ser Humano. Un estudio bioestratinómico de los yacimientos arqueológicos de Olduvay (Tanzania)", Munibe, 51, 123-136.

ESTÉVEZ, J., 1979: La Fauna del Pleistoceno catalán, Tesis Doctoral, Universidad Autonoma de Barcelona. Inédita.

ESTÉVEZ, J., 1980: "Aprovechamiento de recursos faunísticos, aproximación a la economía del Paleolítico catalán", Cypsela, 3, 9-30.

ESTÉVEZ, J., 1987: "La fauna de L'Arbreda, en el conjunto de faunas del Pleistoceno Catalán". Cypsela, VI, 73-87.

FERNÁNDEZ LASO, C., 2002: El estudio zooarqueológico y tafonómico de los restos óseos de Macromamíferos del nivel K del Abric Romaní (Capalledes, Barcelona), Tesis de Licenciatura, Departamento de historia y Geografia, Facultad de Letras, Universidad Rovira i Virgili. Inédita.

FERNÁNDEZ PERIS J. y MARTINEZ VALLE R., 1989: "EI Yacimiento del Paleolítico Medio de San Luis (Buñol Valencia)", Saguntum, 22, 11-34.

FERNÁNDEZ PERIS, J., GUILLEN CALATAYUD, P., FUMANAL, M.P. y MARTÍNEZ VALLE, R., 1994: "Cova Bolomor (Tabernes de Valdigna, Valencia). Primeros datos de una secuencia del Pleistoceno Medio", Saguntum, 27, 9-37.

GIBERT J., 1996: "VII Jornadas de Arqueologia Nacional. Murcia", 14-17 Mayo 1996. Museo Arqueológico Municipal (Lorca). Colegio Nacional de Arquitectos.

GUSI, F., CARBONELL, E., ESTÉVEZ, J., MORA R. y MATEO J., 1980: “Avance Preliminar sobre el yacimiento del Pleistoceno Medio del Tossal de la Font. (Villafanes Castellón)", Cuadernos de Prehistoria y Arqueología castellonenses, 7, 7-30.

GUSI, F., GILBER, J., AGUSTI J. y PEREZ CUEVA, A., 1984: "Nuevos datos del yacimiento del Pleistoceno Medio del
Tossal de la Font. (Villafamés Castellón)", Cuadernos de Prehistoria y Arqueología castellonenses, 10, 7-18.

ITURBE, G., FUMANAL, M.P., CARRION, J.S., CORTELL, E., MARTÍNEZ, R., GUILLEN, M.P., GARRALDA, M. P. y VANDERMERSCH, B., 1993: "Cova Beneito (Muro Alicante). Una perspectiva interdisciplinar", Recerques del Museu D'Alcoi, II, 23-88.

LINDLY, J., 1988: "Hominid and Carnivore activity at Middle and Upper Paleolithic cave sites in Eastern Spain", Munibe, 40, 45-70.

MAREAN, C.W., 1998: "A Critique of the evidence for scavenging by Neandertals and early modern humans: New data from Kobech Cave (zagros mountains, Iran), Die Kelders Cave 1 layer 10 South Africa". Journal of Human Evolution, 35, 111-136.

MARTÍN PENALA, 1986:"Los grandes mamiferos del yacimiento pleistocénico superior de Cueva Horá", Antropología y Paleoecologia Humana, 4, 102-129.

MARTÍNEZ VALLE, R., 1996: Fauna del Pleistoceno Superior en el País Valenciano. Aspectos económicos, huellas de manipulación y valoración paleoambiental, Tesis Doctoral, Universidad de Valencia. Inédita.

MONTES BERNÁRDEZ, R., 1985: "Excavaciones en la Cueva Perneras (Lorca)", Noticiario Arqueológico Hispánico, 23, 7-59.

MONTES BERNÁRDEZ, R., 1988: "Restos malacológicos y paleoecológicos del Musteriense de la Cuesta de Murca (Sureste de España)", Anales de Prehistoria y Arqueologia, 4, 27-32.

MONTES BERNÁRDEZ, R., 1991: "Consideraciones generales sobre el Musteriense en el Sur y Suroeste español (Murcia, Albacete y Andalucia)", Verdolay, 4, 7-13.

MORA, R., TERRADAS, X., MARTÍNEZ, J., PRADOS, E.y PALLARÉS, A., 1992: "Primera aproximación al estudio de las ocupaciones humanas en la cueva de L'Estret de Tragó. Os de Balaguer, Lleida", en Utrilla, P. (ed).: Aragón/ Litoral Mediterráneo. Intercambios Culturales durante la Prehistoria. Ponencias y Comunicaciones.

PÉREZ RIPOLL, M., 1977: Los mamiferos del yacimiento Musteriense de Cova Negra, Trabajos Varios del Servicio de Investigación Prehistórica, 53.

ROSELL J., 2001 Patró d'aprovisonament de biomasses animals durant el Pleistocé Inferior I Mig (Sierra de Atapuerca, Burgos) i Superior (Abric Romani, Barcelona), Tesis Doctoral, Departamento de Historia y Geografia, Facultad de Letras, Universidad Rovira i Virgili. Inédita.

RUEDA, J.M., 1993: L'acció antrópica sobre les matèries dures animals durant el Pleistocé del Nord-est de Catalunya, Tesis Doctoral, Universidad de Girona. Inédita.

RUEDA, J.M. y TERRADAS, X., 1997: "Cova 120: Un exemple des activités de subsistence au Paléolithique Moyen dans les Pyrénées Orientales", XVIII Rencontres internationale d'Archéologie et d'histoire d'Antibes, 349361.

RUIZ BUSTOS, 1978. "Edad y estudio faunístico del yacimiento kárstico de las Yedras», Estudios Geológicos, 34 , 323-334.

SARRIÓN, l., 1990: "El Yacimiento del Pleistoceno Medio de la Cova del Corb (Ondara Alicante)", Archivo de Prehistoria Levantina, XX, 43-75.

SERNA LÓPEZ, J. L., 1997: "Consideraciones sobre economía y ocupación del territorio durante la prehistoria inicial. El caso de los yacimientos Paleolíticos y Epipaleolíticos de la cuenca del Río Mundo", Archivo de Prehistoria Levantina, XXII, 57-71. 
STINER, M., 1994: Honor Amoung Thieves: A Zooarchaeological study of Neandertal ecology, Princeton University Press, Princeton.

TORO MOYANO, I. y ALMOLLANA GALLEGO, M., 1981: "Cueva Colomela I (Caparacena Granada) Nuevo yacimiento Musteriense en el Prau de la Hoz", Cuaderno de Prehistoria de la Universidad de Granada, 6, 9-15.

VILLAVERDE, V. y MARTÍNEZ VALLE, R., 1992: "Economía y aprovechamiento del Medio en el Paleolítico de la región central del Mediterráneo español", en Romanillo, M. (ed.): Elefantes, ciervos y ovicápridos. Economia y aprovechamiento del Medio en la prehistoria de España y Portugal, 77-95.

VILLAVERDE, V., MARTINEZ, R., GUILLEN, P. M. y FUMANAL, M. P., 1996: "Mobility and the role of the small game in the Middle Paleolithic of the Central region of the Spanish Mediterranean. A comparison of Cova Negra with other Paleolithic deposits". en Carbonell, E. y Vaquero, D.: The last neandertals the first anatomically Modern Humans, 267-288.

YRAVEDRA SÁINZ DE LOS TERREROS, J. 2000: "Patrones de Representación Anatómica; una hermenéutica equivocada", Arqueoweb, 3. htpp//www.ucm.es/info/ arqueoweb/

YRAVEDRA SÁINZ DE LOS TERREROS, J. 2001: Zooarqueológica de la Península Ibérica. Implicaciones Tafonómicas y Paleoecológicas en el debate de los homínidos del Pleistoceno Superior, BAR International Series 979, Oxford. 